\title{
INTRODUCTION TO THE ISSUE
}

At this time of accountability, cutbacks, layoffs, and down-sizing, it has become more important than ever to provide evidence to substantiate program impact, effectiveness, and efficiency. To that end, agencies and service providers must provide evidence through the use of systematic methods to determine the extent to which a program is achieving its goals and purposes. Program evaluation addresses a wide range of questions from whether the program is reaching its target and is being implemented properly to questions of fiscal accountability, evaluability, effectiveness with respect to what it was intended to do, and effectiveness with respect to benefits versus cost.

Program evaluation then can be considered as a twofold process: to assess the program's effectiveness and to evaluate its accountability. Within the area of effectiveness are two basic types of evaluation: formative, which looks at a program in progress to determine if there are areas that should be revised, deleted, etc.; and summative, which looks at a program at the end to ask the "Did it work?" question. Regarding the issue of accountability, the most often used evaluation method is cost-benefit or cost-effectiveness analysis.

Although some approach program evaluation with fear and trepidation, when conducted appropriately and with the program and its beneficiaries in mind, it can be both an opportunity to repair weaknesses and reward strengths. The Roberts and Roberts article provides a discussion of the need and rationale for program evaluation along with some evaluation models. In addition, this article outlines some implications for preservice and inservice training of agency personnel.

The Garske, Trach, and Leung article describes several evaluation models that can be utilized with vocational rehabilitation programs. They further review current practices used for evaluation by some state and federal vocational rehabilitation agencies and some of the implications of these practices.

The Thornton article addresses the internal and external validity and generalizability issues with respect to benefit cost analyses. To further illustrate these issues, Thornton compared the Illinois and Virginia Commonwealth studies of supported employment.
The Liebert and Weissman article also examines an evaluation method, specifically the use of the comparison groups design when evaluating transition programs. Their article provides some examples of studies that use true experimental and quasi-experimental control group designs to control for most potential threats to internal validity and allow one to attribute outcomes accurately.

Hill and Ruth provide a comprehensive comparison of the relationship between quality and cost in addition to financial incentives in evaluating the impact of supported employment programs. They further present an example of the use of interagency quality teams to increase quality and reduce costs. Finally they discuss the use of the CQI (Continuous Quality Improvement) model as an instrument for evaluating programs with a focus on quality relative to cost.

Wood and Steere also describe in detail an instrument that can be utilized to evaluate supported employment programs. They provide information regarding both a process of evaluation and a series of standards by which to evaluate programs. Their standards are based on diverse quality measurement systems and a series of guiding principles for evaluating programs.

Salzberg et al. present a quantitative study conducted to compare salaries, benefits, responsibilities, etc. of rehabilitation personnel with those of special educators. This study provides an example of a normative evaluation of factors that impede rehabilitation programs from providing services: staff retention, motivation, and longevity/stability.

While providing a comparison of some existing evaluation tools, the article by Kregel also presents the critical programmatic and policy issues that must be incorporated into any evaluation effort. He further outlines complexities necessary to access strengths and weaknesses of programs.

These articles cover a range of perspectives, and they address issues that arise when looking at the need, implication, and concerns related to program evaluation in vocational rehabilitation. For evaluation to be a beneficial and integral part of vocational rehabilitation programs, it must be a systematic, valid, timely, and thorough examination of a program and its components. It must have the support of all persons involved and take

$J$ Vocat Rehabil 1992; 2(2):7-8

Copyright (C) 1992 by Andover Medical Publishers, Inc. 
into account factors that may be external to the program but that still have an impact on it. It must provide information that will increase our knowledge of the strengths and weaknesses of our programs so that we can make them better. We hope the information provided, examples given, and models described will serve as useful tools in achieving this goal.

Barbara Helms, PhD

Issue Editor 